\title{
An analysis of three-dimensional elasto-plastic sinusoidal contact
}

\author{
Vijaykumar Krithivasan ${ }^{\mathrm{a}}$ and Robert L. Jackson ${ }^{\mathrm{a}}$ \\ ${ }^{a}$ Department of Mechanical Engineering, Auburn University, Auburn, AL 36849, USA
}

Received 21 November 2006; accepted 22 January 2007; published online 6 March 2007

\begin{abstract}
Researchers have developed many models to simulate the elasto-plastic contact of spheres. However, there does not appear to exist a closed-form analytical model for elasto-plastic three-dimensional sinusoidal contact. This work uses a finite element model (FEM) to characterize elasto-plastic sinusoidal contact. Although at initial contact the sphere and sinusoidal case are very similar and can both be described by the classic elastic Hertz contact case, once the contact is pressed past a certain range of deformation the two cases are very different. The model produces equations which can be used to approximately relate the area of contact to the contact pressure for elastoplastic sinusoidal contact. The equations are fit to the FEM results and existing elastic solutions of sinusoidal contact. An empirical expression for the average pressure which causes complete contact between elasto-plastic sinusoidal contacts is also provided.
\end{abstract}

KEY WORDS: contact mechanics, asperity, elastic-plastic contact

Nomenclature

$a$

$A$

B

C

$d$

$e_{y}$

E

$f$

F

$h$

$H$

$p^{*}$

$p$ *

$p_{\text {ep }}{ }^{*}$

$\bar{p}$
$R$

$S_{y}$

$\Delta$

$\lambda$

$v$

Subscripts

$c$

ep

JGH

KE radius of the area of contact for single peak (whendeformation is small)

area of contact

material dependent exponent

critical yield stress coefficient

material and geometry dependant exponent

yield strength to elastic modulus ratio, $S_{y} / E$

elastic modulus

spatial frequency (reciprocal of wavelength) contact force

height of sinusoidal surface from base

hardness

average contact pressure

average pressure for complete contact

average pressure for complete contact (elasto-

plastic)

average pressure over entire surface

radius of curvature of tip of sinusoidal surface

orhemispherical asperity

yield strength

amplitude of sinusoidal surface

wavelength $(1 / f)$

Poisson's ratio

interference between hemisphere and surface

critical value at onset of plastic deformation elasto-plastic

from model by Johnson et al. [1]

from model by Kogut and Etsion [2]
*To whom correspondence should be addressed.
E-mail: robert.jackson@eng.auburn.edu

\section{Introduction}

Computational modeling of contact between surfaces, or contact mechanics, has been realized as an important field for many years, probably starting sometime around the classic solution of elastic sinusoidal contact developed by Hertz in 1888 (originally developed to model optical contacts) [3]. Since then the field has evolved to consider rough surface contact between mechanical devices and recently in MEMS. Many earlier models employed mostly spherical contact models to simulate individual asperity peaks on a surface. Several used alternative approaches to model periodic surface roughness as sine waves $[1,4]$. These first models considered only elastic contact. However, due to the high loads often seen in contact situations, the stresses can cause plastic deformation as well. The FFT based contact methodology outlined by Stanley and Kato [5] made use of these elastic contact models to model rough surface contact by first transforming the surface data into the frequency domain.

Elasto-plastic sinusoidal contact has recently become more important with the development of several multiscale contact models [6-12]. A model of elasto-plastic sinusoidal contact would be very useful to these multiscale contact models. Gao et al. [13] modeled twodimensional sinusoidal contact using the finite element method, and made note of some interesting results which will also be considered in the current analysis. The current work finds trends similar to [13] resulting from the three-dimensional case.

The current analysis will examine the case of threedimensional elastic perfectly plastic sinusoidal contact by building on these previous works, using fundamental solid mechanics theory, and conducting a parametric study using the finite elements method. The 
sinusoidal surface considered by the current work is described by

$$
h=\Delta \cdot\left(1-\cos \left(\frac{2 \cdot \pi \cdot x}{\lambda}\right) \cos \left(\frac{2 \cdot \pi \cdot y}{\lambda}\right)\right)
$$

and is shown in figure 1 , where $h$ is the height of the sinusoidal surface from its base. This is very similar to the surface used by Johnson et al. [1] and results in the same analytical equations for elastic contact (as will be discussed later).

\subsection{Spherical contact models}

Although the following section may at first seem off subject, in reality spherical contact is very useful for modeling sinusoidal contact, especially when the deformation is restricted to the tips of the peaks (see figure 2). For this reason, Johnson et al. [1] provide two limiting solutions to three-dimensional sinusoidal contact, and the first being based upon the Hertz elastic spherical contact solution. The Hertz solution provides closedform expressions to the deformations and stresses of two spheres in a purely elastic contact. The two spheres may have different radii and different elastic properties. However, the closed-form solutions render an equivalent case where a single elastic sphere, having an equivalent elastic modulus, $E^{\prime}$, and an equivalent radius, $R$, is in contact with a rigid flat (see equations (2-5) that follow). The interference, $\omega$, can be described as the distance the sphere is displaced normally into the rigid flat. The Hertz solution assumes that the interference is small enough such that the geometry does not change significantly. The solution also approximates the sphere surface as a parabolic curve with an equivalent radius of curvature at its tip. The resulting equations for contact radius and load from the Hertz solution are:

$$
\begin{gathered}
A_{E}=\pi R \omega \\
F_{E}=\frac{4}{3} E^{\prime} \sqrt{R}(\omega)^{3 / 2}
\end{gathered}
$$

where

$$
\begin{gathered}
\frac{1}{E^{\prime}}=\frac{1-v_{1}^{2}}{E_{1}}+\frac{1-v_{2}^{2}}{E_{2}} \\
\frac{1}{R}=\frac{1}{R_{1}}+\frac{1}{R_{2}}
\end{gathered}
$$

and $E_{1}, v_{1}, R_{1}, E_{2}, v_{2}, R_{2}$, are the elastic properties and radii of sphere 1 and 2, respectively.

Again, since the sinusoidal surface and the tip of a sphere are very similar at initial contact, the initiation of plastic deformation (defined as the critical interference) can be derived from Hertz contact theory, but can also be used for sinusoidal contact in for cases of relatively small loads and interferences. The critical values of interference, area, and load are derived for the case of initial sinusoidal contact (when the contact geometry is still similar to a parabola or sphere) in Appendix 1.

Kogut and Etsion [2] performed a finite element analysis of the case of an elastic-perfectly plastic sphere in contact with a rigid flat. Their work gives a very detailed analysis of the stress distribution in the contact region, and piecewise empirical expressions are provided for the contact area, the contact force, and the average contact pressure. At values $\omega / \omega_{c}<1$ the Hertz contact

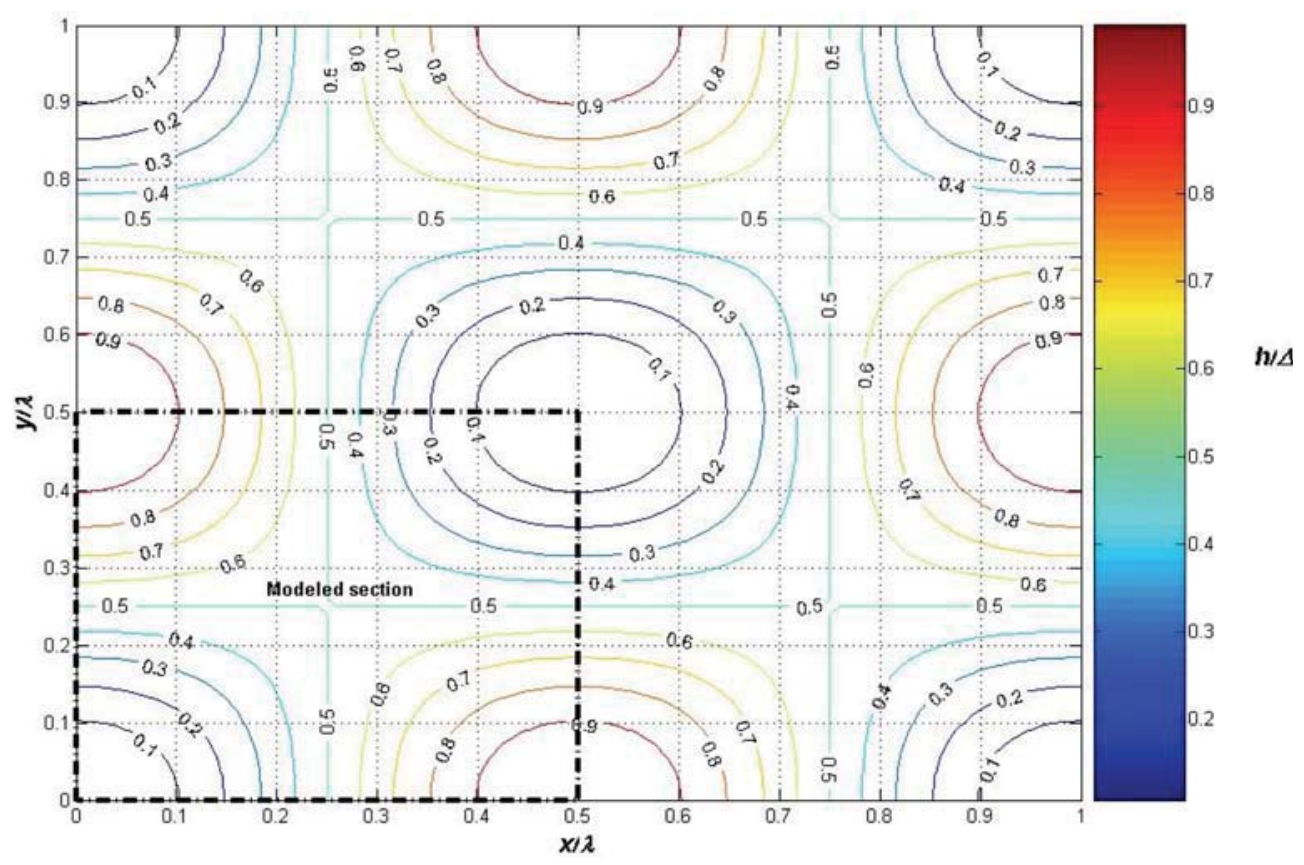

Figure 1. Topographical contour plot of the sinusoidal surface geometry. 


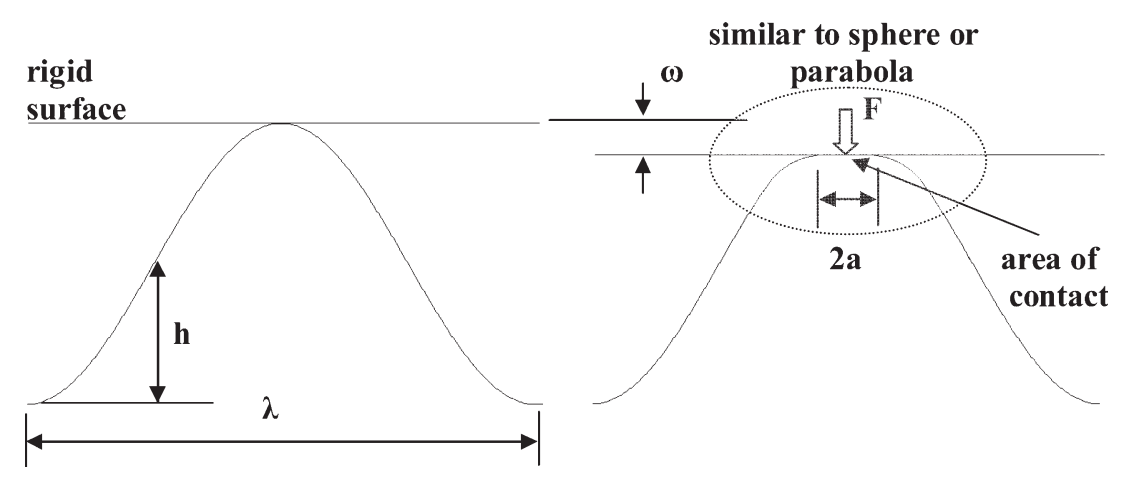

Figure 2. Cross-section of sinusoidal type contact.

solution is assumed. Two sets of equations are provided for the ranges $1<\omega / \omega_{c}<6$, and $6<\omega / \omega_{c}<110$. They describe the deformation only up to $\omega / \omega_{c}=110$, at which point full plasticity is assumed. In their analysis, the value of hardness, $H$, is set to be fixed at $2.8 \cdot S_{y}$. Jackson and Green [14] also provide a more complicated model which may describe large deformations better than the Kogut and Etsion (KE) model. However, Quicksall et al. [15] found that the Jackson and Green and KE model agree fairly well for small deformations and the KE model is also much easier to algebraically manipulate. For these reasons, the KE model is used in the current work to model the initial elasto-plastic deformation of the sinusoidal surfaces.

The KE model is used in the current work to obtain an empirical relationship between contact pressure and area. Since the current work is mostly concerned with spherical contact for small load and interferences, the pressure-interference and the area-interference relation's for $1<\omega / \omega_{c}<6$ from Kogut and Etsion [2] are used:

$$
\begin{aligned}
\left(\frac{p}{S_{y}}\right) & =1.19\left(\frac{\omega}{\omega_{c}}\right)^{0.289} \\
\left(\frac{A_{K E}}{A_{c}}\right) & =0.93\left(\frac{\omega}{\omega_{c}}\right)^{1.136}
\end{aligned}
$$

With some manipulation equation (6) becomes

$$
\frac{\omega}{\omega_{c}}=\left[\frac{1}{1.19}\left(\frac{p}{S_{y}}\right)\right]^{\frac{1}{0.289}}
$$

The average contact pressure, $p$, is related to the average pressure, $\bar{p}$, over the sinusoidal surface by:

$$
p=\left(\frac{\bar{p}}{2 \cdot A_{K E}} \lambda^{2}\right)
$$

The $\bar{p}$ denotes average pressure over the entire surface, including areas that are not in contact while $p$ denotes average contact pressure, just in the contact area. When complete contact occurs, $\bar{p}=p$. Substituting equations (8) and (9) into equation (7) the following is obtained:

$$
\frac{A_{K E}}{A_{c}}=\left(\frac{\bar{p}}{2 \cdot A_{K E} \cdot S_{y} \cdot 1.19} \lambda^{2}\right)^{3.93}
$$

Further solving for $A_{K E}$ results in the equation below:

$$
A_{K E}=\left(A_{c}\right)^{\frac{1}{4.93}}\left(\frac{\bar{p}}{2.38 \cdot S_{y}} \lambda^{2}\right)^{\frac{3.93}{4.93}}
$$

An equation similar to equation (11) is used in the current work to model initial elasto-plastic contact of the sinusoidal surfaces. This is explained in more detail later.

\subsection{Elastic sinusoidal contact}

The analysis for the case of 3-D waviness developed by authors Johnson, Greenwood and Higginson [1] (hereafter referred to as "JGH") provides a relation between pressure and contact area. First $\bar{p}$ is defined as the average pressure on the surface (considering both contacting and non-contacting regions) and $p^{*}$ is the amplitude of a sinusoidal pressure superimposed on the mean pressure that yields complete contact. Complete contact is defined as when the entire sinusoidal surface is flattened and in contact with the opposing surface (i.e., there is no gap between the surfaces). The amplitude of the pressure profile, $p^{*}$ is given as:

$$
p^{*}=\sqrt{2} \pi E^{\prime} \Delta f
$$

where $E^{\prime}$ is the reduced elastic modulus, $\Delta$ is the amplitude of the sinusoidal surface, and $f$ is the frequency or reciprocal of wavelength, $\lambda$. Thus when $\bar{p} p *$, the pressure loads the surfaces so that there is no gap between them. Alternatively, when $\bar{p}<p *$ the contact is not complete, and a closed-form solution for the three-dimensional waviness contact problem is not available. However, Johnson et al. [1] provides two asymptotic solutions to the problem. For $\bar{p}<<p *$ the following equation derived from Hertz contact theory applies:

$$
\left(A_{\mathrm{JGH}}\right)_{1}=\frac{\pi}{f^{2}}\left[\frac{3}{8 \pi} \frac{\bar{p}}{p *}\right]^{2 / 3}
$$


and when $\bar{p}$ approaches $p^{*}$ (i.e., contact is nearly complete) the following equation applies:

$$
\left(A_{\mathrm{JGH}}\right)_{2}=\frac{1}{f^{2}}\left(1-\frac{3}{2 \pi}\left[1-\frac{\bar{p}}{p *}\right]\right)
$$

Since no general analytical solution is available, an equation linking equations (13) and (14) is fit by Jackson and Streator [6] to the experimental and numerical data provided by Johnson et al. [1]:

For $\frac{\bar{p}}{p *}<0.8$ :

$$
A=\left(A_{\mathrm{JGH}}\right)_{1}\left(1-\left[\frac{\bar{p}}{p *}\right]^{1.51}\right)+\left(A_{\mathrm{JGH}}\right)_{2}\left(\frac{\bar{p}}{p *}\right)^{1.04}
$$

For $\frac{\bar{p}}{p *} \geq 0.8$

$$
A=\left(A_{\mathrm{JGH}}\right)_{2}
$$

\subsection{Elasto-plastic sinusoidal contact}

The previous cases of elasto-plastic spherical contact and elastic sinusoidal contact are now sufficiently expanded using finite element results so that a model of elasto-plastic sinusoidal contact can be formulated. Since it has been established that at low deformations the sinusoidal contact will behave similarly to the spherical contact, this work will now move onto the case of heavy deformations in sinusoidal contact.

Intuitively the hardness of a sinusoidal shaped contact will follow a much different trend than the spherical case. As shown in the figure 3, the slope of the surface at the edge of contact will be much different for the spherical case as the amount of deformation is increased. While the spherical case approaches a rod or cone type problem $[14,16]$, the sinusoidal case appears to reduce to a flat against a flat problem as interference increases and the sinusoidal surface is flattened out. Thus, it would appear that the hardness of the sinusoidal surface will initially stay constant or slightly decrease with interference (depending on the value of $\Delta / \lambda$ ) and then eventually start increasing again as flattening occurs. For sinusoidal surfaces with large values of $\Delta / \lambda$, the hardness to yield strength ratio $\left(H / S_{y}\right)$ will probably decrease initially because the geometry is very similar to that of a post or rod. However, for smaller values of $\Delta / \lambda, H / S_{y}$ will probably never decrease because the surface is more flat and the post or rod shape is never approached. Then, when the contact is more flat, the effective $H / S_{y}$ becomes larger (as shown in the following sections). It should be noted that the surfaces simulated in this work are for fairly low values of $\Delta / \lambda$, and so examples of the first case are never seen.

When the sinusoidal surface is almost completely flattened, one may initially conclude that the contact condition is similar to the spherical case at low $a / R$ values and thus $H / S_{y}$ returns to approximately 2.84 or higher values (as was concluded by Gao et al. [13]). However, if this case is examined closely, it becomes unclear what the actual limiting pressure is during the complete contact case. For example, once complete contact has occurred, the case becomes very similar to that of a compressed cavity of material (see figure 4). In this case the material cannot deform in the lateral direction parallel to the surface because there are neighboring identical sinusoidal contacts. During elastic contact and using Hooke's Law the normal pressure is related to the lateral stresses by

$$
\sigma_{\text {lateral }}=\frac{1-3 v}{1-v} P
$$
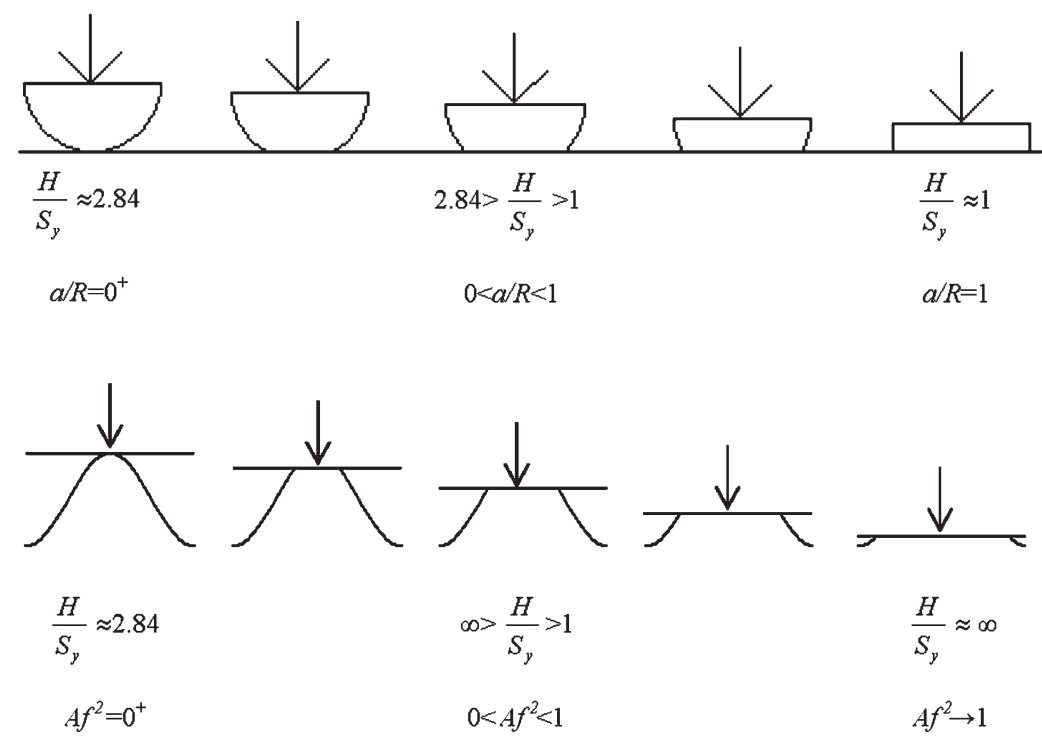

Figure 3. Diagram of the hypothetical progression of change in hardness with geometry for spherical and sinusoidal contact. 


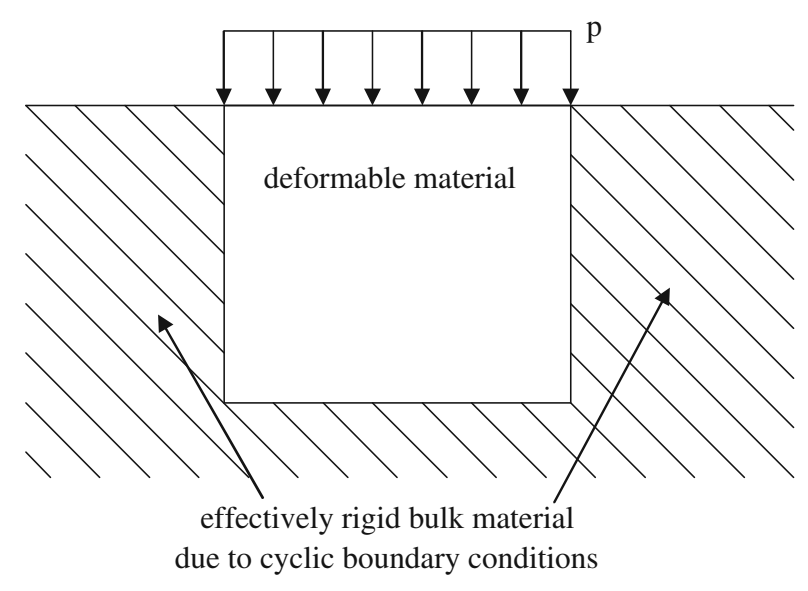

Figure 4. Compressed cavity of material.

However, the current case is not elastic, but it is unclear what the material does when large plastic deformations occur, or if it can reach the plastic regime once complete contact has occurred. One way to model a plastically deforming material is to assume that it conserves volume $(v=0.5)$. For the cavity case (shown in Figure 4 and described by equation (17)), plastic deformation then cannot occur since the shape of the material is restricted, and so the only way for the material to deform is for it to compress volumetrically (hydrostatic stress). Thus the volume of material can only deform elastically and $H / S_{y}$ approaches infinite values (see figure 3). In the work by Gao et al. [13] and Gao and Bower [10] it also appears that $H / S_{y}$ increases dramatically when a loaded 2-D surface approaches the case of complete contact. However, in the current 3-D model the authors' find that $p^{*}$ during fully plastic deformation is not limited by a value of $5.8 \cdot S_{y}$ that appears to limit the 2-D case [13].

Greenwood and Rowe [17] cover the topic of plastic crushing of serrated surfaces and also note that the force to cause complete contact becomes drastically larger as the surfaces come closer together. The case of a conical indenter correlates to this case as well, since as the angle of the cone tip approaches $180^{\circ}$ the stress beneath the indenter will then become hydrostatic (see Marsh [18] and Johnson [19]). This is also a similar concept to that employed by the fractal contact models of Majumdar and Bhushan [20], in that they predict as the contact area increases, the contacting asperities will effectively become more elastic. However, it should be noted that in reality the material in the current case did initially undergo severe plastic deformation, so that even though the contact appears to behave elastically near complete contact, it has undergone a large amount of plastic deformation to arrive at that condition.

\section{Finite element model (FEM)}

A three-dimensional model was developed and the commercial ANSYS $^{\mathrm{TM}} 8.1$ package was used to further analyze the elasto-plastic sinusoidal contact problem. Owing to the symmetry of the sinusoidal surface (shown in figures 1 and 5), only a quarter section of the whole problem is modeled. The sinusoidal surface was then pressed against a rigid flat surface. In all, more than 42,000 elements were used in the analysis. Solid45, which is an 8-node brick element, was used within the entire volume of model. Contac174 and Target170 elements collectively formed the contact pair to model interaction between the surfaces.

The number of elements was increased iteratively by a factor of 2 until mesh convergence was obtained. The final value corresponded to concordant values over two successive iterations. Upon final convergence the rigid flat surface, comprising of Contac174 elements, was a uniform mesh formed by a $21 \times 21$ array of elements. In all, there were approximately 1200 contact elements in the model. The contact stiffness value was also altered until the model agreed with the elastic solution (see verification section below).

The uniform mesh on the rigid surface is used to predict the real contact area with the sinusoidal surface. By determining the contact status of each node during post-processing the total number of nodes in contact and the corresponding contact pressures are obtained from the nodal solution for incremented values of $\bar{p} / p_{\mathrm{ep}}^{*}$. The ratio of the number of nodes in contact to the total number of nodes over the surface gives the real area of contact normalized by the apparent or nominal area of contact.

The displacement method was employed to simulate the contact problem. This method applies a finite displacement on the rigid flat surface in the $z$-direction toward the sinusoidal surface, and then solves the contact problem. Displacement boundary conditions were enforced on the surface areas of the solid model, and not in the contact area (see figure 5). The bottom surface was fixed in all directions. Constraints were applied to

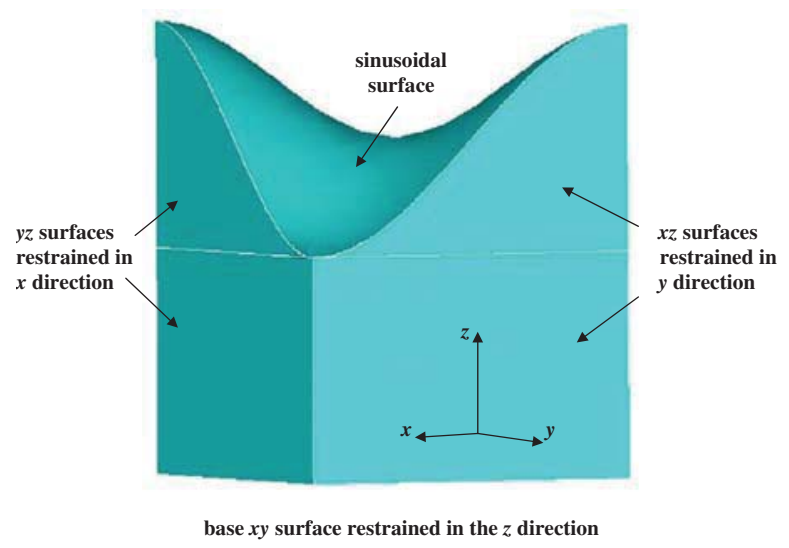

Figure 5. Schematic of degree of freedom restraints used for the one quarter sinusoidal FEM model (actual modeled geometry is much longer in the $\mathrm{z}$ direction). 


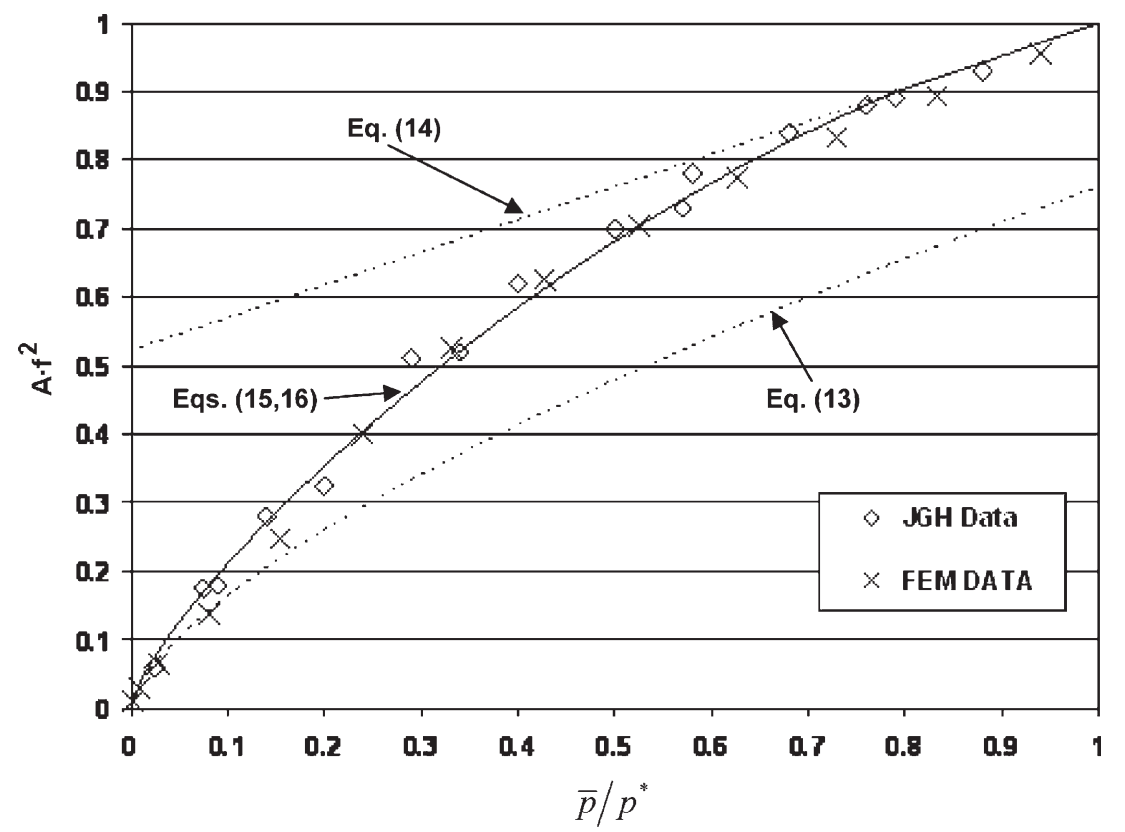

Figure 6. Comparison of elastic FEM results with JGH model.

surfaces located on the $x y, x z$, and $y z$ planes in the direction perpendicular to the plane (thus enforcing a cyclic boundary condition). For example, a surface area along the $y z$ planes was constrained in the $x$-direction, as shown in figure 5 .

\section{Verification of model accuracy}

Model accuracy was achieved by comparing the elastic results of the finite element model (FEM) to that of Johnson et al.'s data [1] (see figure 6). As shown, the curves for both the data sets followed almost identical paths. An average error of $6 \%$ was found to exist between the FEM and JGH data over the entire contact range.

\section{Elasto-plastic FEM results}

The material properties of the solid 3-D model used to run the elastic case, were modified for the elastoplastic case. To enhance convergence, as outlined by Brizmer et al. [21], the material of the sinusoidal surface was assumed elasto-plastic bi-linear isotropic hardening with a yield stress, $S_{y}$, of $1 \mathrm{GPa}$ and a tangent modulus, $E_{T}, 2 \%$ of the Young's modulus $E$. This isotropic hardening significantly improves the convergence without causing a significant change in the results.

In order to formulate a fit for the FEM contact pressure a parametric analysis of the elasto-plastic sinusoidal surface contact problem was conducted. A benchmark case was set to analyze the contact problem. The material properties used for the benchmark case are $E=200 \mathrm{GPa}, S_{y}=1 \mathrm{GPa}$, and $v=0.3$. The dimensionless geometric ratio $\Delta / \lambda$ was set as 0.02 . The material properties along with the $\Delta / \lambda$ ratio were then individually varied to perform a parametric study.

First, a range of yield stresses were considered in the model (see figures 7 and 8). The yield strength was varied from $S_{y}=0.75 \mathrm{GPa}$ to $S_{y}=2.25 \mathrm{GPa}$. First, the results are presented as a plot of the average pressure divided by the yield strength, $\bar{p} / S_{y}$, as a function of the normalized area, $A f^{2}$ (see figure 7). As the area increases the contact is becoming more complete and the amount of deformation is increasing. Traditionally this would be considered the fully plastic regime and the average pressure, $\bar{p}$, would be the hardness, $H$. As shown, the ratio of $\bar{p} / S_{y}$ increases past the typical $H / S_{y}$ value of 3 and even past the value of 5.8 found in [13] for 2-D sinusoidal surfaces in contact. This agrees with the earlier theory that the contact becomes more elastic as the contact area becomes more complete $\left(A f^{2} \rightarrow 1\right)$.

Next, the average contact pressure, $\bar{p}$, resulting from the elasto-plastic model was normalized using $p^{*}$. Then the normalized contact area $A f^{2}$ was plotted versus $\bar{p} / p *$. It can be seen that the normalized contact area increases steadily as the yield strength decreases. It can be seen from figure 8 that for a $\bar{p} / p *=0.42$, the normalized contact area ratio is 1 for all the cases (the contact is complete). Thus for the elasto-plastic cases, complete contact occurs much earlier than when it occurs in elastic contact, as is intuitively expected. Although the trend captured partially resembles the perfectly elastic behavior, in order to find empirical expressions for the elasto-plastic case the findings 


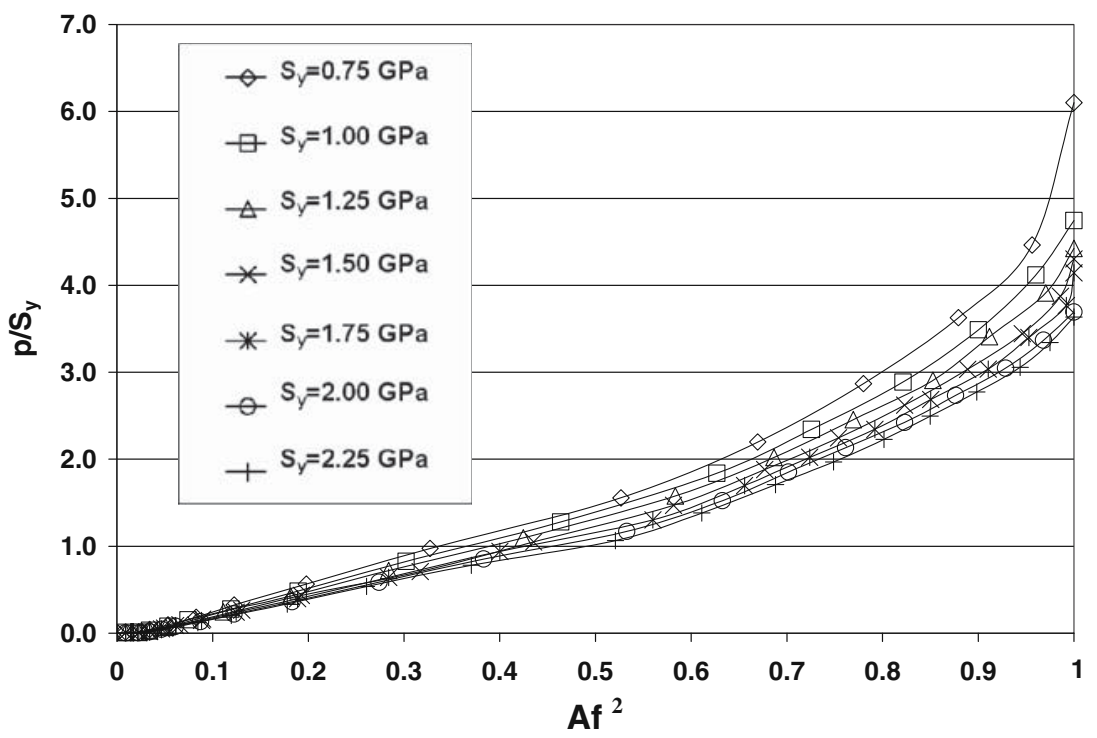

Figure 7. $p / S_{y}$ versus contact area ratio $\left(A f^{2}\right)$ for different yield strengths.

suggest that a new average pressure to cause complete contact during elasto-plastic contact, $p_{\text {ep }}^{*}$, can be found.

The average pressure, $p_{\text {ep }}^{*}$, that causes complete contact is extracted from the finite element model data for each modeled case. This value corresponds to the average pressure when the area ratio, $A / f^{2}=1$. At this stage complete contact occurs between the rigid flat and the sinusoidal surface. An equation is fit to the values of $p^{*}$ ep from the finite element data. The goal of the empirical fit is to obtain a single expression that takes into consideration both the material and geometric properties of the elasto-plastic sinusoidal contact problem that is modeled. Since the material properties and geometric properties are each varied independently from the benchmark case, an equation can easily be fit to each trend. It was also found that the effects of $S_{y}$ and $E^{\prime}$ appear to be almost exactly inverse and can be combined into one normalized variable $S_{y} / E^{\prime}$. The resulting equation fit to the FEM data is given as

$$
\frac{p_{\mathrm{ep}}^{*}}{p^{*}}=\left(4.172 \cdot \frac{S_{y}}{E^{\prime}}+0.0173\right) \cdot \sqrt{\frac{\lambda}{\Delta}}
$$

The effect of $S_{y} / E^{\prime}$ on $p^{*}$ ep $/ p^{*}$ is shown by the plot in figure 9 . The relationship of $p^{*}$ ep $/ p^{*}$ to $S_{y} / E^{\prime}$ appears to be linear in nature. As shown in figure 9, equation (18) differs from the FEM data by an average error of $3.82 \%$

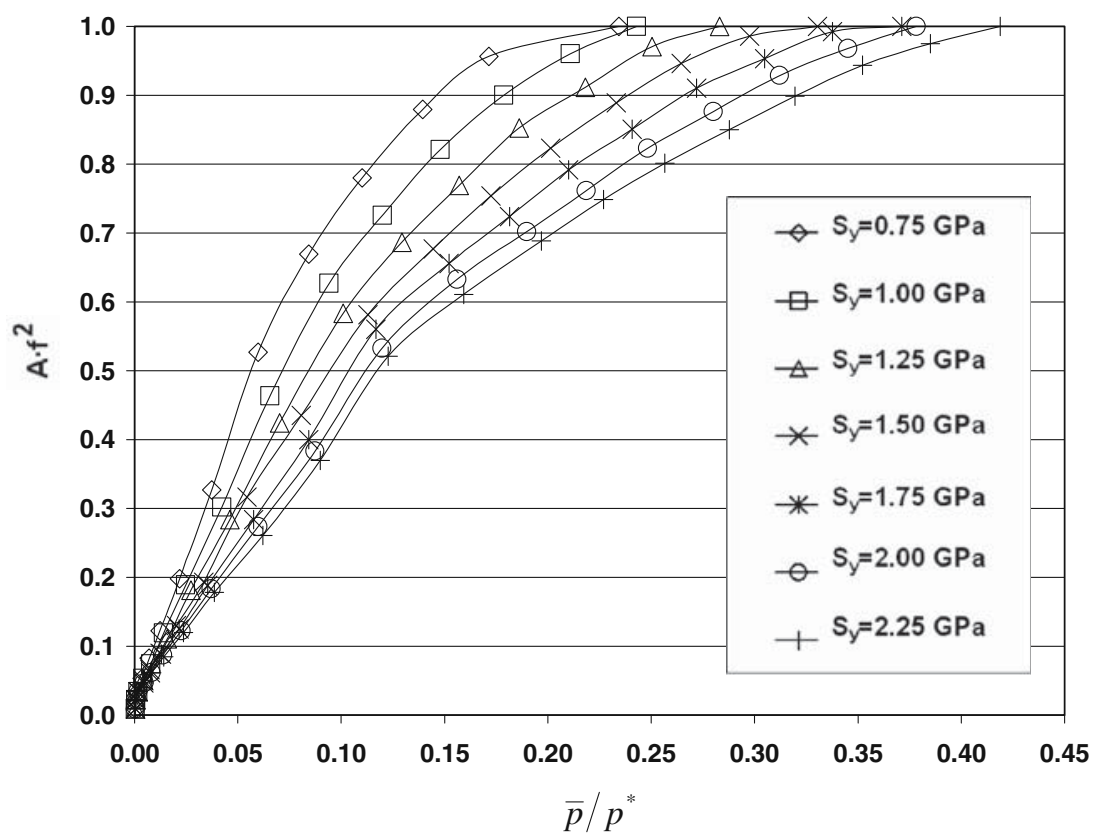

Figure 8. Contact area ratio $\left(A f^{2}\right)$ versus $\bar{p} / p^{*}$ for different values of yield strength. 


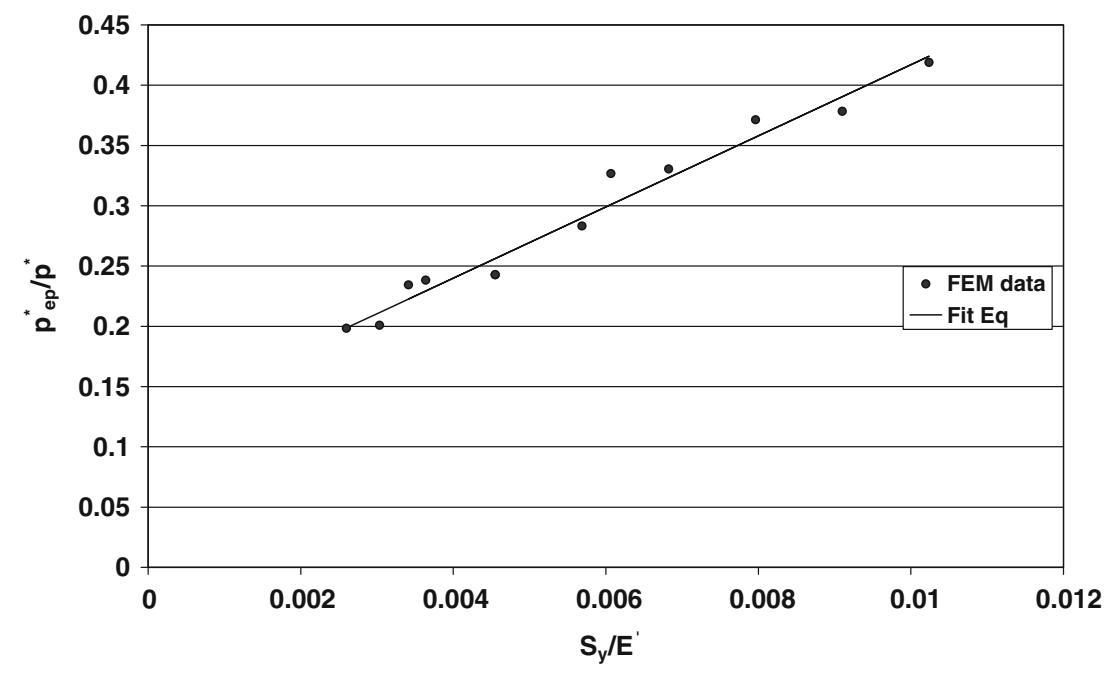

Figure 9. The effect of $S_{y} / E^{\prime}$ on pressure to cause complete contact (equation (18)).

with the maximum and minimum errors being $7.89 \%$ and $0.17 \%$, respectively. The empirical fit generated thus seems to be effective.

The effect $\Delta / \lambda$ has on $p_{\mathrm{ep}}^{*} / p^{*}$ appears to be nonlinear, as shown in figure 10 . The $p^{*}$ ep $/ p^{*}$ value also decreases with an increase in the $\Delta / \lambda$ ratio. As apparent in equation (18), a square root function appears to produce a reasonable fit. The average error resulting from the fit is $2.72 \%$, with maximum and minimum error values of $5.56 \%$ and $0.23 \%$, respectively.

Next the $p_{\text {ep }}^{*}$ values obtained from equation (18) are used as a normalization factor for the contact pressure, $\bar{p}$, predicted by the elasto-plastic FEM model (see figures 11-13). This normalization is useful because it is successful at collapsing the curves onto almost the same curve (see figure 11 in comparison to figure 8). Since similar plots are not shown for the results when
$E$ and $\Delta / \lambda$ are varied, the values of $\bar{p} / p^{*}$ varied by as much as $100.5 \%$ between the $E$ cases and $91.1 \%$ between the $\Delta / \lambda$ cases, which is large in comparison to the error values given above. The results for the cases of various yield strengths, $S_{y}$, are shown in the plot of $A f^{2}$ versus $\bar{p} / p_{\mathrm{ep}}^{*}$ in figure 11 . In this case the equivalent elastic modulus, $E^{\prime}=220 \mathrm{GPa}$ and the geometric property $\Delta / \lambda=0.02$ are held constant for all the cases modeled. From a lower $S_{y}$ value of $0.75 \mathrm{GPa}$ to a higher value of $2.25 \mathrm{GPa}$ the values of curves seem to increase slightly. As the yield strength, $S_{y}$, increases the curves appear to converge to a single curve. Towards complete contact it can be seen that although the curves are for different yield strengths, they converge to the same point due to the normalization of $\bar{p}$ by $p_{e p}^{*}$. Since the trend of the normalized contact area $A f^{2}$ as a function of $\bar{p} / p_{\mathrm{ep}}^{*}$ should always begin at $A f^{2}=0$,

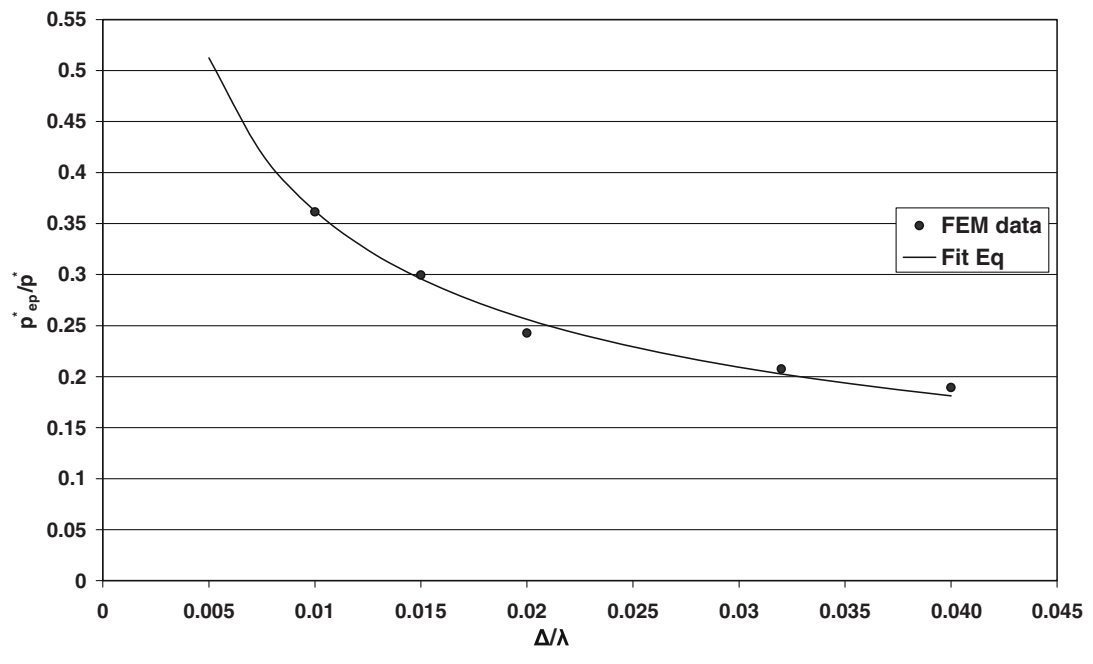

Figure 10. The effect of $\Delta / \lambda$ on pressure to cause complete contact (equation (18)). 


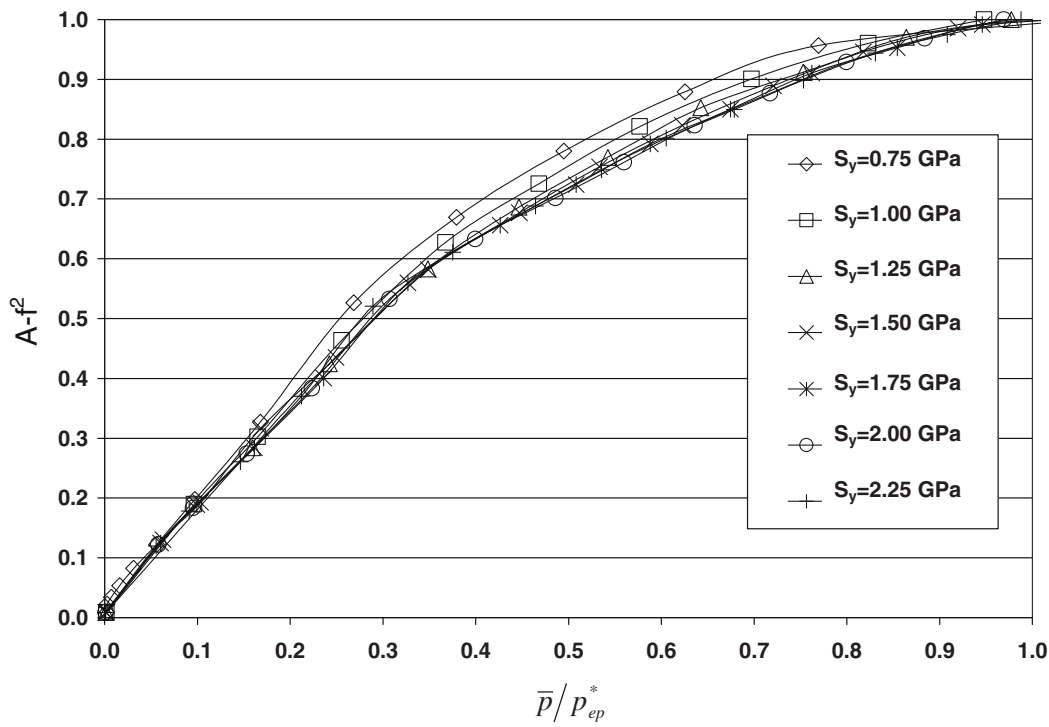

Figure 11. Contact area ratio $\left(A f^{2}\right)$ versus $\bar{p} / p_{\mathrm{ep}}^{*}$ for different yield strength values.

$\bar{p} / p_{\mathrm{ep}}^{*}=0$ (just before initial contact) and end at $A f^{2}$ $=1, \quad \bar{p} / p_{\mathrm{ep}}^{*}=1$ (when complete contact occurs), the normalization is successful just at collapsing these end points together. However, the curvature of the normalized trend can still depend on the material and geometric properties. Therefore, additional measures are taken to fit equations to the FEM data.

From figure 12, it is seen that the elastic modulus $E^{\prime}$ and the yield strength $S_{y}$ both display similar trends. This case is modeled by varying only the equivalent elastic moduls, $E^{\prime}$, while the yield strength, $S_{y}=1 \mathrm{GPa}$ and $\Delta / \lambda=0.02$ are held constant for all the different cases. As the elastic modulus increases, the curve also increases. At lower values of $\bar{p} / p_{\mathrm{ep}}^{*}$ the curves seem to fall onto a single curve. When the $\bar{p} / p_{\mathrm{ep}}^{*}$ value reaches 0.1 the curves seem to diverge. Near complete contact the curves converge again.

The geometric property $\Delta / \lambda$ is now varied from 0.01 to 0.04 , as shown in figure 13 . The equivalent modulus $E^{\prime}=220 \mathrm{GPa}$ and the yield strength, $S_{y}=1 \mathrm{GPa}$, are held constant in these various cases that are modeled. The contact pressure, $\bar{p}$, from the FEM data is again normalized by the average pressure for complete elastoplastic contact, $p_{\text {ep. }}^{*}$ This ratio is then plotted versus the contact area ratio. It can be seen that normalized area $\left(A / A_{n}\right)$ steadily increases when the $\Delta / \lambda$ ratio increases. Another point should be noted, that the lower values of the ratio $\Delta / \lambda$ allow for the solution to converge faster,

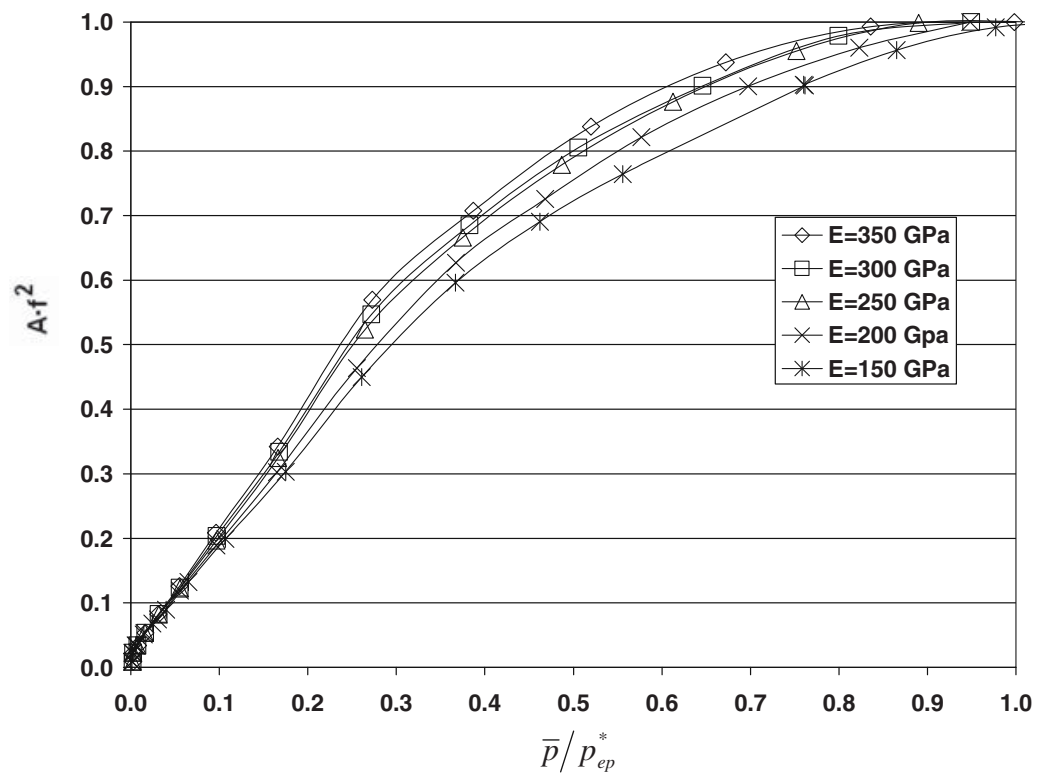

Figure 12 Contact area ratio $\left(A f^{2}\right)$ vs. $\bar{p} / p_{\mathrm{ep}}^{*}$ for different values of Young's modulus E. 


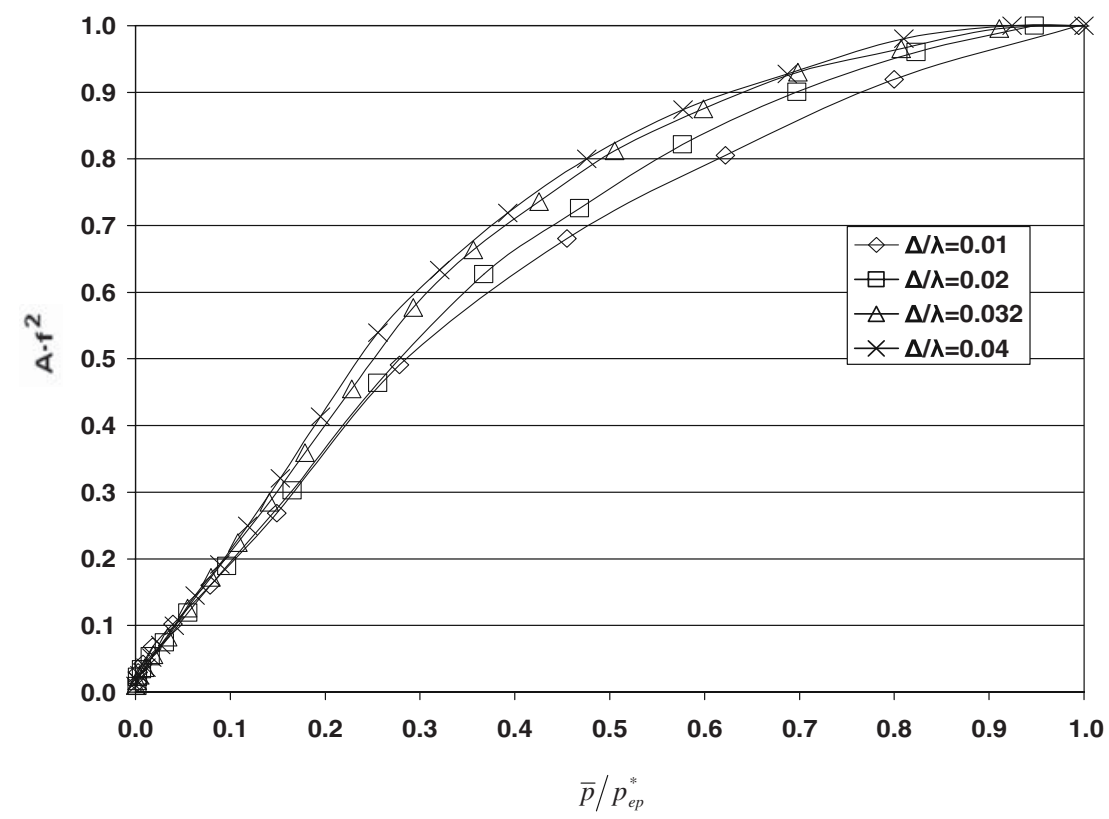

Figure 13. Contact area ratio $\left(A f^{2}\right)$ vs. $\bar{p} / p_{\mathrm{ep}}^{*}$ for different values of the dimensionless geometric quantity $\Delta / \lambda$.

while some higher values of $\Delta / \lambda$ do not converge at all. The reason for this dependence on $\Delta / \lambda$ for convergence could be from the fact that with higher $\Delta / \lambda$ ratios larger deformations are required to flatten out the sinusoidal surface.

The area described by the KE model is next modified to better suit the current elasto-plastic sinusoidal contact problem. The premise for the argument is based on the fact that when $A / A_{c}=1$, the pressure to critical pressure ratio $p / p_{c}$ should equal 1 . Notice that $p$ is now used to denote the average contact pressure over the contact area, while $\bar{p}$ denotes the average pressure over the entire surface area. Based on the KE model, a logical function to model initial elasto-plastic contact is described below

$$
\frac{A}{A_{c}}=\left(\frac{p}{p_{c}}\right)^{d}
$$

where $p_{c}$ from equation (A5) in Appendix 1 is

$$
p_{c}=\left(\frac{2 \cdot C \cdot S_{y}}{3}\right)
$$

substituting this into equation (19) we get,

$$
\frac{A}{A_{c}}=\left(\frac{p}{2 \cdot C \cdot S_{y}} \cdot 3\right)^{d}
$$

where average pressure over the contact area, $p$, in equation (20) is converted to the average pressure over the entire surface, $\bar{p}$, by

$$
p=\left(\frac{\bar{p} \cdot \lambda^{2}}{2 \cdot A}\right)
$$

By substituting in equation (21) into equation (20) the following expression results

$$
\frac{A}{A_{c}}=\left(\frac{\bar{p} \cdot \lambda^{2} \cdot 3}{4_{y} \cdot C \cdot A}\right)^{d}
$$

Solving for $A$ in equation (22), one obtains the modified expression for contact area, which is now termed as $A_{\mathrm{ep}}$. Also noting that the two sphere-like peaks occur in the $\lambda^{2}$ area of sinusoidal contact than a factor of two must be included. The result is given by

$$
A_{e p}=2\left(\frac{A_{c}}{2}\right)^{\frac{1}{1+d}}\left(\frac{3 \cdot \bar{p}}{4 \cdot C \cdot S_{y}} \lambda^{2}\right)^{\frac{d}{1+d}}
$$

Although this expression (equation (23)) for area is derived differently, it is interesting to note that it is a similar result to the area to pressure relation from the $\mathrm{KE}$ model (equation (11)). The value of $d$ is obtained empirically by fitting a curve to the FEM results. To allow a good fit with the FEM results, the value of $d$ found in equation (23) for $A_{\text {ep }}$ is allowed to vary with $E^{\prime} / S_{y}$ and $\Delta / \lambda$. The expression for $d$ is given by

$$
d=C_{1} \cdot\left(\frac{E^{\prime}}{S_{y}} \cdot \frac{\Delta}{\lambda}\right)^{C_{2}}
$$

where, $C_{1}=3.8$ and $C_{2}=0.11$ and are constants which are obtained empirically. $E^{\prime}$ and $S_{y}$ are the effective Young's modulus and yield strength, respectively. For the benchmark case of $E^{\prime}=220 \mathrm{GPa}$, 


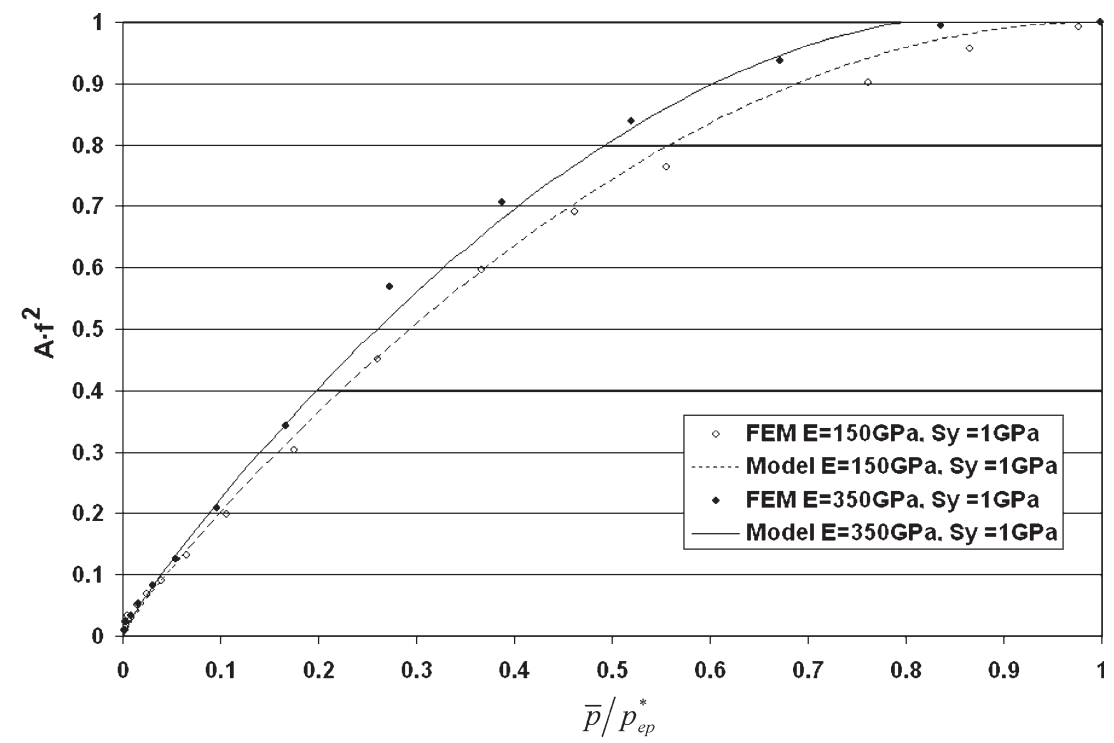

Figure 14. Comparison of the FEM results and the elasto-plastic sinusoidal contact model (marked by lines) given by equation (25) for different yield strength values.

$S_{y}=1 \mathrm{GPa}$, and $\Delta / \lambda=0.02$, then $d=4.47$. As expected, this is close to the value of 4.93 that is predicted by the KE model.

To model the contact area as a function of load, the results of JGH are now modified with the elasto-plastic results now given by equations (18 and 23). The modified version of the model to consider elasto-plastic deformation is

$$
A=\left(A_{\mathrm{ep}}\right)\left(1-\left[\frac{\bar{p}}{p_{\mathrm{ep}}^{*}}\right]^{1.51}\right)+\left(A_{\mathrm{JGH}}\right)_{2}\left(\frac{\bar{p}}{p_{\mathrm{ep}}^{*}}\right)^{1.04}
$$

where $A_{\mathrm{ep}}$ is the spherical elasto-plastic contact area as predicted by the model given by equations ( 23 and 24 ) and based on the KE model. Then as the load increases, the contact will diverge from the spherical case and asymptotically approach the sinusoidal case. Since the $\mathrm{H} / \mathrm{S}_{y}$ will theoretically increase as the contact becomes more complete (see previous discussion concerning figure 4), the contact area may theoretically approach the elastic $\operatorname{limit}\left(A_{\mathrm{JGH}}\right)_{2}$ as $\bar{p} / p_{\mathrm{ep}}^{*}$ approaches 1 . However, when the solution approaches $\left(A_{\mathrm{JGH}}\right)_{2}$ the average pressure is $p_{\mathrm{ep}}{ }^{*}$ instead of $p^{*}$ as in the elastic case. Also, if $A$, as predicted by equation (25), increases past $\lambda^{2}$ then $A=\lambda^{2}$.

The FEM results are presented alongside the model given by equation (25) in figures 14-16. In figure 14 we see the fit between the two extreme cases that were

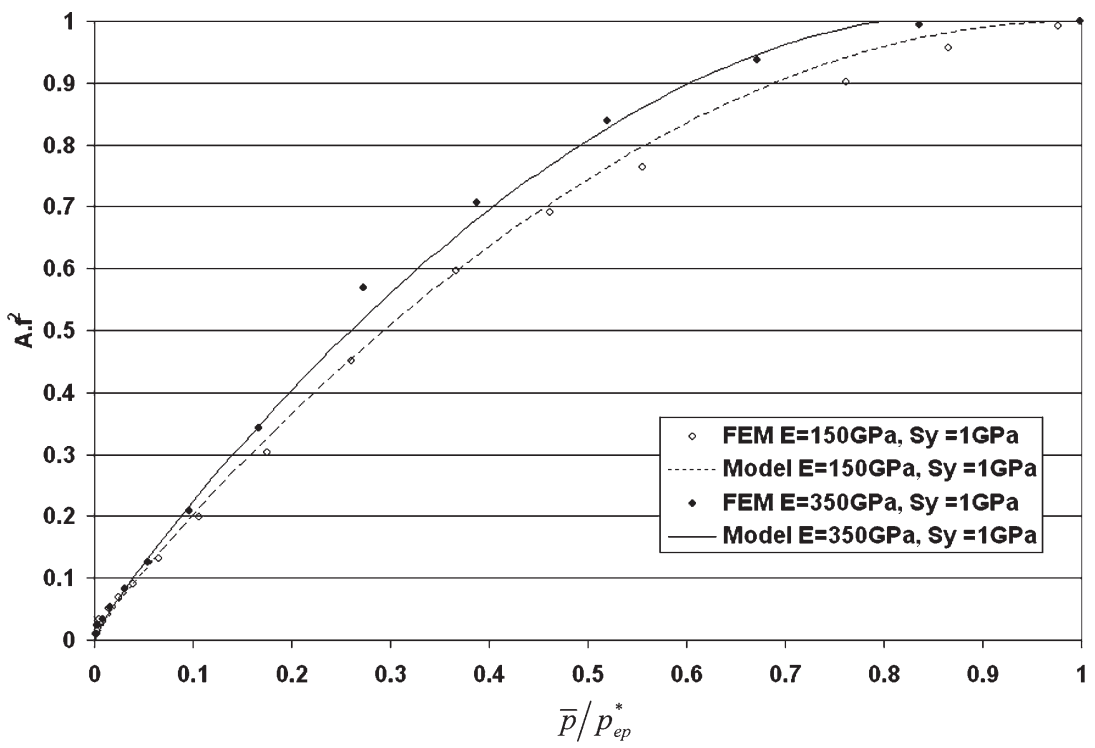

Figure 15. Comparison of the FEM results and the elasto-plastic sinusoidal contact model (marked by lines) given by equation (25) for different Young's modulus values. 


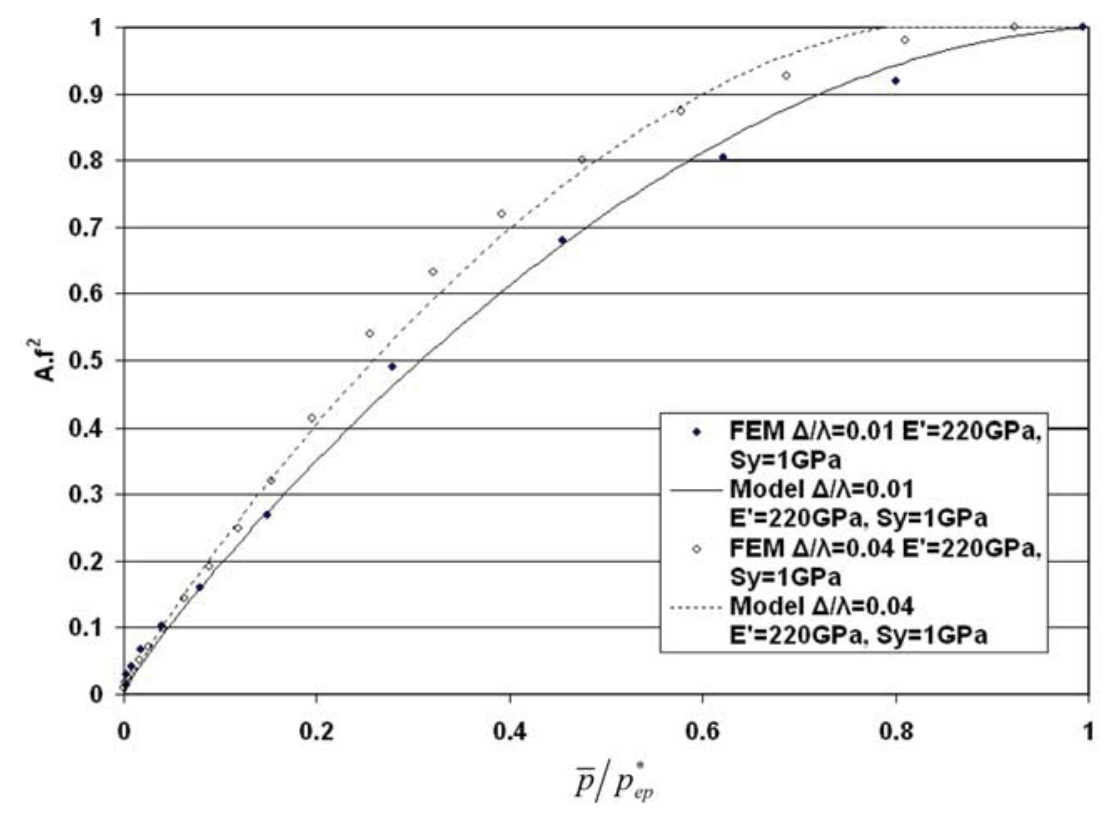

Figure 16. Comparison of the FEM results and the elasto-plastic sinusoidal contact model (marked by lines) given by equation (25) for different sinusoidal geometries.

modeled based on the yield strength of the material. The lowest $S_{y}$ value considered being $0.75 \mathrm{GPa}$ and the higher value being $2.25 \mathrm{GPa}$. We can see clearly that the modified KE model for contact area agrees fairly well with the numerical results. The average error between the new model given by equation (25) and the FEM results when the yield strength is varied independently is less than $5 \%$. Likewise, the plots in figures 15 and 16 show that the model also compares well for the cases where the elastic modulus, $E$, and the geometry, $\Delta / \lambda$, are varied (the error in these cases is also less than $5 \%$ ).

\section{Conclusions}

The contact problem comprised of an elasto-plastic deformable sinusoidal surface and a rigid flat was analyzed and modeled by the finite element method. The numerical analysis of the current finite element model provided an approximate solution for the elasto-plastic regime of the sinusoidal surface. An empirical equation based on previous sinusoidal elastic contact models and elasto-plastic spherical contact models was formulated to fit the FEM data. Dimensionless expressions were empirically derived for the mean contact pressure which causes complete contact during large elasto-plastic deformations. By normalizing the contact area and the mean contact pressure, the current model provides analytical expressions for the elasto-plastic regime of contact area as a function of pressure. The expression for the contact area for sinusoidal contact problem was obtained by modifying the Kogut and Etsion model [2] and the elastic sinusoidal models [1]. The errors in the numerical fits are fairly low (less than $5 \%$ ) and suggest that fits are reasonable. These equations should prove useful for modeling contact between rough periodically structured surfaces and in multiscale rough surface contact models.

\section{Appendix 1: Derivation of critical values for sinusoidal contact}

The critical or initial interference to cause yielding can be derived independently of hardness as

$$
\omega_{c}=\left(\frac{\pi \cdot C \cdot S_{y}}{2 E^{\prime}}\right)^{2} R
$$

where $C$ is given by

$$
C=1.295 \exp (0.736 v),
$$

and $v$ is the Poisson ratio of the material that has the lowest value of $C \cdot S_{y}$, and thus yields first. The critical force, $F_{c}$ is then calculated at the critical interference, $\omega_{c}$, to be

$$
F_{c}=\frac{4}{3}\left(\frac{R}{E^{\prime}}\right)^{2}\left(\frac{C}{2} \pi \cdot S_{y}\right)^{3}
$$

Similarly, the critical contact area is

$$
A_{c}=\pi^{3}\left(\frac{C S_{y} R}{2 E}\right)^{2} .
$$


By dividing the critical force (equation (A3)) by the critical area (equation (A4)) the critical contact pressure is defined as

$$
p_{c}=\left(\frac{2 C S_{y}}{3}\right) .
$$

These critical values can be modified for use in the sinusoidal case considered in this work by substituting in the relation

$$
R=\frac{1}{4 \pi^{2} \Delta f^{2}},
$$

which is the radius of curvature for the tip of a sinusoidal surface. In addition there are actually two spherelike peaks of contact which occur during a $\lambda^{2}$ area of sinusoidal contact (see figure 1). This results in a modified critical force given by

$$
F_{c}=\frac{1}{6 \pi}\left(\frac{1}{\Delta f^{2} E^{\prime}}\right)^{2}\left(\frac{C}{2} \cdot S_{y}\right)^{3} .
$$

Similarly, the modified critical contact area is

$$
A_{c}=\frac{2}{\pi}\left(\frac{C S_{y}}{8 \Delta f^{2} E^{\prime}}\right)^{2} \text {. }
$$

Since $R$ does appear in equation (A5), the critical pressure is not influenced by $R$. It should be noted that equations (A5, A7, and A8) are only valid for the initial stages of contact between sinusoidal surfaces (when the contacting surfaces are geometrically similar to spheres). However, these critical values are useful in determining when a sinusoidal contact begins to deforming in the elasto-plastic range.

\section{References}

[1] K.L. Johnson, J.A. Greenwood and J.G. Higginson, Int. J. Mech. Sci. 27(6) (1985) 383

[2] L. Kogut and I. Etsion, J. Appl. Mech. Trans. ASME 69(5) (2002) 657.

[3] K.L. Johnson, Contact Mechanics (Cambridge University Press, 1985).

[4] H.M. Westergaard, ASME J. Appl. Mech. 6 (1939) 49.

[5] H.M. Stanley and T. Kato, J. Tribol. Trans. ASME 119(3) (1997) 481.

[6] R.L. Jackson and J.L. Streator, Wear 261(11-12) (2006) 1337.

[7] C.K. Bora, E.E. Flater, M.D. Street, J.M. Redmond, M.J. Starr, R.W. Carpick and M.E. Plesha, Tribol. Lett. 19(1) (2005) 37.

[8] M. Ciavarella, G. Demelio, J.R. Barber and Y.H. Jang, Proc. R. Soc. Lond. A. 456 (2000) 387.

[9] M. Ciavarella, G. Murolo, G. Demelio and J.R. Barber, J. Mech. Phys. Solids 52(6) (2004) 1247.

[10] Y.F. Gao and A.F. Bower, Proc. R. Soc. A. 462 (2006) 319.

[11] B.N.J. Persson, Phys. Rev. Lett. 87(11) (2001) 116101.

[12] B.N.J. Persson, F. Bucher and B. Chiaia, Phys. Rev. B. 65 (2002) 184106.

[13] Y.F. Gao, A.F. Bower, K.S. Kim, L. Lev and Y.T. Cheng, Wear 261(2) (2006) 145.

[14] R.L. Jackson and I. Green, ASME J. Tribol. 127(2) (2005) 343.

[15] J.J. Quicksall, R.L. Jackson and I. Green, IMechE J. Eng. Trib. Part J. 218(4) (2004) 313.

[16] M.M. Chaudhri, I.M. Hutchings and P.L. Makin, Philos. Mag. 49(4) (1984) 493.

[17] J.A. Greenwood and G.W. Rowe, J. Appl. Phys. 36 (1965) 667.

[18] D.M. Marsh, Proc. R. Soc. A. 279 (1964) 420.

[19] K.L. Johnson, J. Mech. Phys. Solids 18 (1970) 115

[20] A. Majumdar and B. Bhushan, ASME J. Tribol. 113(1) (1991) 1.

[21] V. Brizmer, Y. Zait, Y. Kligerman and I. Etsion, J. Mech. Mater. Struct. 1(5) (2006) 865 